\title{
BMJ open How long after a miscarriage should women wait before becoming pregnant again? Multivariate analysis of cohort data from Matlab, Bangladesh
}

To cite: DaVanzo J, Hale L, Rahman M. How long after a miscarriage should women wait before becoming pregnant again? Multivariate analysis of cohort data from Matlab, Bangladesh. BMJ Open 2012;0:e001591. doi:10.1136/bmjopen-2012001591

- Prepublication history and additional material for this paper are available online. To view these files please visit the journal online (http://dx. doi.org/10.1136/bmjopen2012-001591)

Received 9 June 2012 Accepted 29 June 2012

This final article is available for use under the terms of the Creative Commons Attribution Non-Commercial 2.0 Licence; see http://bmjopen.bmj.com

For numbered affiliations see end of article

Correspondence to

Dr Julie DaVanzo;

julie@rand.org

\section{ABSTRACT}

Objective: To determine the optimum interpregnancy interval (IPI) following a miscarriage.

Design: Multivariate analysis of population-based, prospective data from a demographic surveillance system. Setting: Pregnancies in Matlab, Bangladesh, between 1977 and 2008.

Participants: 9214 women with 10453 pregnancies that ended in a miscarriage and were followed by another pregnancy outcome.

Main outcome measures: Outcome of pregnancy following the miscarriage was singleton live birth, stillbirth, miscarriage or induced abortion. For pregnancies that ended in live birth: early neonatal, late neonatal and postneonatal mortality.

Results: Compared with IPIs of 6-12 months, pregnancies that were conceived $\leq 3$ months after a miscarriage were more likely to result in a live birth and less likely to result in a miscarriage (adjusted relative risk ratio (RRR) $0.70,95 \% \mathrm{Cl} 0.57$ to 0.86 ) or induced abortion $(0.50,0.29$ to 0.89$)$. Induced abortions were significantly more likely following IPIs of 18-24 months (2.36, 1.48 to 3.76$), 36-48$ months (2.73, 1.50 to 4.94$)$, and $>48$ months (3.32, 1.68 to 2.95$)$, and miscarriages were more likely following IPIs of 12-17 months (1.25, 1.01 to 1.56$)$ and $>48$ months $(1.90,1.40$ to 2.58$)$. No significant effects of IPI duration are seen on the risks of a stillbirth. However, IPIs $\leq 3$ months following a miscarriage are associated with significantly higher late neonatal mortality for the infant born at the end of the IPI (adjusted hazard ratio (HR) 1.74, 1.06 to 2.84), and IPIs of 12-18 months are associated with a significantly lower unadjusted risk of postneonatal mortality $(0.54,0.30$ to $0.96)$.

Conclusions: The shorter the IPI following a miscarriage, the more likely the subsequent pregnancy is to result in a live birth. However, very short IPIs may not be advisable following miscarriages in poor countries like Bangladesh because they are associated with a higher risk of mortality for the infants born after them.

\section{INTRODUCTION}

Many studies have assessed the effect on mater$\mathrm{nal}^{1}$ and perinatal ${ }^{2}$ outcomes and on infant

\section{ARTICLE SUMMARY}

Article focus

- To assess the association between the duration of the interpregnancy interval following a miscarriage and the outcome of the next pregnancy in Matlab, Bangladesh.

\section{Key messages}

- The shorter the interpregnancy interval (IPI) following a miscarriage, the more likely the subsequent pregnancy is to result in a live birth.

- However, very short IPIs ( $\leq 3$ months) following a miscarriage are associated with a higher risk of late neonatal mortality for the infants born after them.

- Hence, IPIs $\leq 3$ months following a miscarriage may not be advisable in poor countries like Bangladesh.

Strengths and limitations of this study

- Study considers data from a poor, developing area -rural Bangladesh. Most previous studies of this topic have been of industrialised countries.

- Sample size (10 453) is larger than that in most studies of this topic, though not as large as in a recent study of Scottish women.

- Study considers mortality during infancy in addition to pregnancy outcomes.

- Study considers effects of even shorter and even longer intervals than previously considered.

- Data on pregnancy outcomes were carefully collected and likely to be of high quality, but probably not as high quality as clinical data.

and child mortality ${ }^{3}$ of pregnancy spacing following a live birth or following a live birth or stillbirth. However, very few studies have sought to identify the optimum interpregnancy interval (IPI) following a miscarriage (spontaneous abortion); the studies that have been done are generally of women living in industrialised countries, and most have relatively small sample sizes. ${ }^{4-6}$ A recent study ${ }^{7}$ that considered a large sample of women who delivered in Scottish hospitals found that women who 
conceived within 6 months after a miscarriage had better outcomes of the subsequent pregnancy than women who waited longer to conceive again; for example, they were less likely to have a voluntary pregnancy termination (induced abortion) or another miscarriage. In this paper we investigate whether these same findings are seen in a very different setting-among poor women in rural Bangladesh. We also investigate whether infants born at the ends of the intervals died before their first birthday. Women in Bangladesh are more likely to be malnourished than those in industrialised countries, ${ }^{8}$ and hence may be more likely to be nutritionally depleted by a pregnancy, even one that ends in miscarriage.

\section{METHODS}

We use high-quality longitudinal data from the Matlab Demographic Surveillance System (DSS). Matlab is a rural subdistrict of Bangladesh that is well known for its DSS and its Maternal Child Heath-Family Planning (MCH-FP) project, which operates in half of the area covered by the DSS to provide intensive and quality family planning and maternal/child health services. ${ }^{9-11}$

The Matlab DSS contains, for both areas of Matlab, longitudinal records of pregnancy outcomes and deaths for all household members. During their regular visits to each household, fortnightly between 1966 and 1999, monthly between 2000 and 2006, and bimonthly since 2007, the community health workers (CHWs) record pregnancy status at the time of the visit and any pregnancy outcomes or household deaths that occurred prior to the visit.

The DSS provides information on 245091 pregnancies that occurred between 1974 and 2008. In this study we consider the 10435 pregnancies documented in the DSS that began with a miscarriage in January 1977 or later and were followed by another pregnancy outcome (here called the 'focal pregnancy') other than a multiple live birth not later than December 2008. Before 1977, the DSS did not distinguish between spontaneous and induced abortions. In the DSS, a miscarriage (spontaneous abortion) is defined as a spontaneous fetal loss prior to 28 weeks gestation. We exclude from the sample focal pregnancies that ended with multiple live births; 246 pregnancies are excluded for this reason.

We consider the following outcomes of the focal pregnancies that follow the IPI after a miscarriage: singleton live birth, stillbirth, miscarriage and induced abortion. In the DSS, a live birth is the delivery of a live baby at any gestational age; a stillbirth is a fetal loss at 28 weeks or longer gestation; and induced abortion is self-reported. Early-gestation pregnancy termination is legal in Bangladesh if performed in a medical setting before the pregnancy is clinically confirmed. Such pregnancy terminations are done by manual vacuum aspiration by trained female paramedics at the government Health and Family Welfare Centers and are known as 'menstrual regulation' (MR). MR can be performed within 10 weeks of the last menstrual period before pregnancy is clinically confirmed. MR has been available through government and other medical facilities in Bangladesh since the late 1970s, when the government agreed to permit such pregnancy terminations in an effort to replace the practice of unsafe abortion. Pregnancy termination in a non-medical setting or after pregnancy is clinically confirmed is prohibited in Bangladesh except when done to save a woman's life. Our 'induced abortion' category includes both MRs and voluntary pregnancy terminations by other means. (Since 1989, when method of pregnancy termination was first distinguished in the DSS, $52 \%$ of terminations have been by MR, $3 \%$ by D\&C and $45 \%$ by other means.)

We also consider mortality of the children born in the focal pregnancies during three subperiods of the first year of life-early neonatal (first week of life), late neonatal (next 3 weeks of life) and postneonatal (the rest of the first year of life). The sample for our analyses of early neonatal mortality is the 8705 IPIs that began with a miscarriage and ended with a live birth. The sample for late neonatal mortality is the 8401 of these that survived the first week of life and were still living in Matlab, and the sample for postneonatal mortality is the 8268 of these that survived the first 4 weeks of life and were still living in Matlab.

The duration of the IPI is defined by measuring the amount of time between the preceding miscarriage and the estimated date of conception of the focal pregnancy. For the 5914 cases for which we know the date of the last menstrual period (DLMP), we estimate the date of conception as occurring 2 weeks after the DLMP before the focal pregnancy. For the 4519 cases for which DLMP was not reported, we estimate the duration of the IPI as the amount of time between the miscarriage and the end of the focal pregnancy less the estimated duration of the focal pregnancy, based on the outcome of that focal pregnancy. Our estimate of pregnancy duration for each type of pregnancy outcome is the average duration of all pregnancies that ended with that outcome for which we know DLMP. These averages are 36 weeks for live births, 33 weeks for stillbirths, 11 weeks for miscarriages and 8 weeks for induced abortions. We have also done all analyses only for the cases for which DLMP was reported; the sizes of the relative risk ratios (RRRs) and hazard ratios (HRs) are similar to those reported here.

Our multivariate analyses control for the woman's age at the time of the focal outcome (with dichotomous indicators for age <20, 20-24, 25-29, 30-34, 35-39 and $\geq 40$ ), the woman's educational attainment and calendar year (approximately 10-year bands of the calendar year of the focal outcome). (We used interactions to explore whether the IPI effects varied over time, but these were never statistically significant.) We also control for the gravidity of the focal pregnancy (dichotomous indicators) and for whether the woman lived in the MCH-FP Area or the Comparison Area of Matlab. Data on maternal age, gravidity, area and calendar year all come from 
the DSS. Information on women's education is from periodic censuses conducted by International Centre for Diarrheal Disease Research, Bangladesh (icddr,b) in the Matlab area. Most of the potential confounders vary significantly with IPI, as can be seen in table 1. Women's ages at both the beginnings and ends of the IPI are positively related to IPI duration, and longer IPIs are more likely to be for higher gravidity and to occur in the later years covered by the data.

\section{Statistical analysis}

We assess the effects of the duration of the IPI on the outcome of the subsequent pregnancy with unadjusted and adjusted RRRs that derive from univariate and multivariate multinomial logistic regressions. The effects of IPI duration on mortality during subperiods of infancy are estimated with Cox proportional hazards models. All models are estimated by Stata 11.0. The hazard model allows for censoring due to moving out of the Matlab area or not completing the at-risk period by the end of 2008. The multivariate analyses control for the variables mentioned above. We used the cluster command in Stata 11.0 to adjust SE for the fact that 1516 women have more than one pregnancy in the sample.

To facilitate comparisons we consider the same categories of IPI durations considered in the recent Love et al study of Scottish women $-\leq 6$ months (0-24 weeks), 6-12, months (25-52 weeks) (reference category), 12-18 months (53-76 weeks), 18-24 months (77-104 weeks) and $>24$ months (105 or more weeks), where each category includes the upper bound but not the lower bound. We also conduct analyses that consider additional categories of IPIs, breaking the $\leq 6$ months category into $\leq 3$ months (0-12 weeks) and 4-6 months (13-24 weeks) to assess the effects of very short intervals, and breaking the $>24$ months category into $24-36,36-48$ and $>48$ months, since other studies have found different effects of such longer intervals. ${ }^{12}$

\section{RESULTS}

The middle of table 2 shows the cross-tabulation of IPI duration and outcome of the focal pregnancy for the IPI categories considered by Love et al. The rows above that show the finer breakdown of the $\leq 6$ months category, and the rows below that show the finer breakdown of the $>24$ months category. Of the 10435 cases in our sample, $4596(44.0 \%)$ conceived $\leq 6$ months after the miscarriage $(20.5 \% \leq 3$ months and $23.5 \%$ in 4 6 months). The next largest percentage is for IPIs of $6-$ 12 months $(28.0 \%)$. The percentages for IPIs of $12-18$ and $18-24$ months are $9.5 \%$ and $6.5 \%$, respectively. IPIs $>24$ months comprise $12.0 \%$ of the sample $(5.5 \%$ are 24-36 months long, $2.8 \%$ are $36-48$ months and $3.7 \%$ are $>48$ months). We find a somewhat higher incidence of short intervals ( $\leq 12$ months) and a somewhat lower incidence of long intervals ( $>24$ months) than

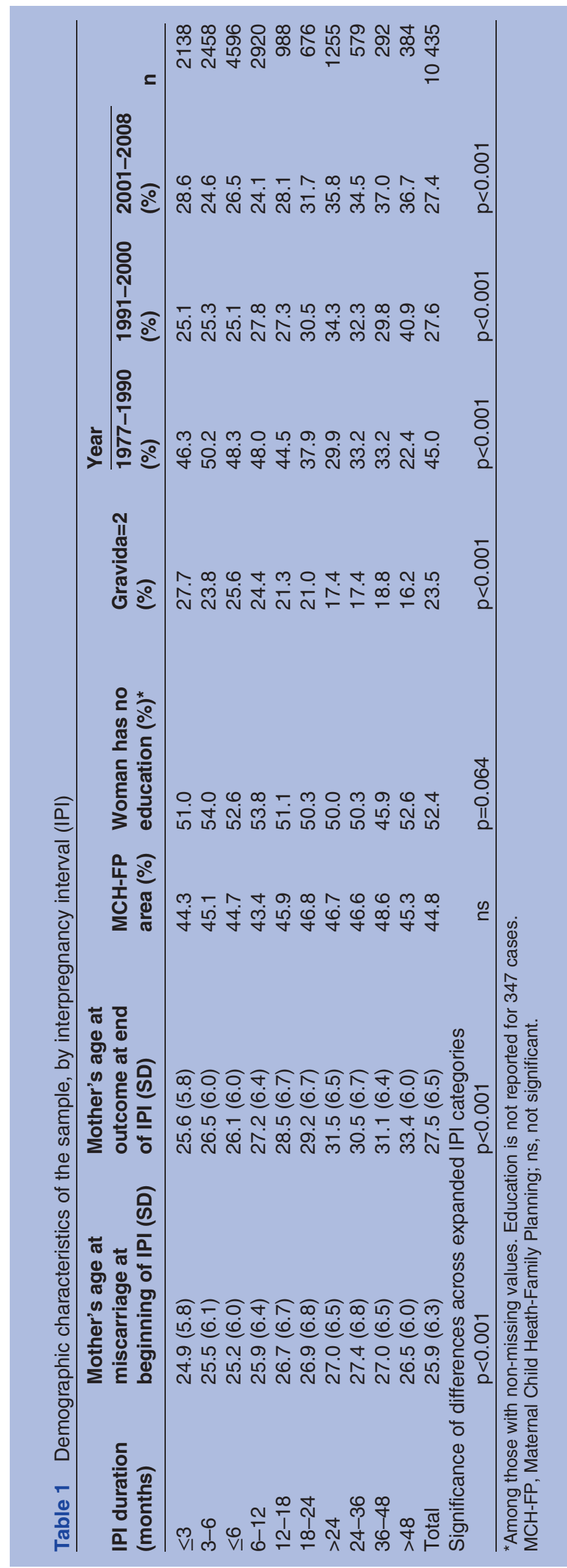


Table 2 Outcomes of subsequent pregnancy after miscarriage in previous pregnancy, by interpregnancy interval (IPI) $(n=10435)$

\begin{tabular}{|c|c|c|c|c|c|c|c|}
\hline \multirow{2}{*}{$\begin{array}{l}\text { IPI duration } \\
\text { (months) (\%) }\end{array}$} & \multicolumn{4}{|c|}{ Outcome of subsequent pregnancy } & \multirow[b]{2}{*}{ Total } & \multirow[b]{2}{*}{ Column \% } & \multirow{2}{*}{$\begin{array}{l}\text { Love et al } \\
\text { column \% }\end{array}$} \\
\hline & Abortion & Miscarriage & Stillbirth & Live birth & & & \\
\hline$\leq 3$ & $16(0.8)$ & $160(7.5)$ & $87(4.1)$ & 1875 (87.7) & 2138 (100.0) & 20.5 & \\
\hline $3-6$ & $33(1.3)$ & $262(10.7)$ & 89 (3.6) & 2074 (84.4) & 2458 (100.0) & 23.5 & \\
\hline$\leq 6$ & 49 (1.1) & $422(9.2)$ & $176(3.8)$ & 3949 (85.9) & 4596 (100.0) & 44.0 & 41.2 \\
\hline$\overline{6}-12$ & $52(1.8)$ & $302(10.3)$ & 114 (3.9) & 2452 (84.0) & 2920 (100.0) & 28.0 & 25.2 \\
\hline $12-18$ & $25(2.5)$ & 125 (12.7) & $45(4.6)$ & 793 (80.3) & 988 (100.0) & 9.5 & 9.6 \\
\hline $18-24$ & $32(4.7)$ & $81(12.0)$ & $20(3.0)$ & $543(80.3)$ & 676 (100.0) & 6.5 & 6.4 \\
\hline$>24$ & $63(5.0)$ & $173(13.8)$ & $51(4.1)$ & $968(77.1)$ & 1255 (100.0) & 12.0 & 17.6 \\
\hline Total & $221(2.1)$ & 1103 (10.6) & 406 (3.9) & 8705 (83.4) & 10435 (100.0) & 100.0 & 100.0 \\
\hline In Love et al & $(4.9)$ & (11.7) & $(0.6)$ & (80.3) & $(97.5)^{\star}$ & & \\
\hline $24-36$ & $15(2.6)$ & $66(11.4)$ & $29(5.0)$ & 469 (81.1) & $578(100.0)$ & 5.5 & \\
\hline $36-48$ & $19(6.5)$ & 38 (13.1) & $9(3.1)$ & 226 (77.9) & $290(100.0)$ & 2.8 & \\
\hline$>48$ & $29(7.6)$ & 69 (18.0) & $13(3.4)$ & $273(71.1)$ & $384(100.0)$ & 3.7 & \\
\hline
\end{tabular}

*The Love et al numbers do not add to $100 \%$ because their data also included ectopic pregnancies ( $0.8 \%$ of all outcomes) and 'other' outcomes $(1.7 \%$ of all outcomes).

Love et al find for Scottish women, but, as seen in the right-hand column of table 2, the IPI distributions are quite similar.

Of all IPIs that began with a miscarriage, $2.1 \%$ ended with an induced abortion, $10.6 \%$ ended with another miscarriage, $3.9 \%$ ended with a stillbirth and $83.4 \%$ ended with a live birth (table 2). The percentage of postmiscarriage pregnancies that end with a live birth decreases as the length of the IPI increases. It is highest for the shortest IPIs $\quad(85.9 \%$ for $\quad \mathrm{IPI} \leq 6$ months and $87.7 \%$ for IPI $\leq 3$ months) and lowest for the longest IPIs $(77.1 \%$ for IPI $>24$ months and $71.1 \%$ for IPI $>48$ months). The percentages for induced abortion and miscarriage each increase nearly monotonically as IPI increases, but there is little systematic pattern for stillbirths. A similar pattern was found for Scottish women, as can be seen in table 2, though the incidence of stillbirth is lower in their data and the incidence of induced abortion higher than we find for Matlab, Bangladesh.

Of all IPIs that began with a miscarriage and ended with a live birth, 292 of those live-born children died in their first week of life (33.5 early neonatal deaths per
1000 live births). Of those who survived the first week, $13.1 / 1000$ died in the next 3 weeks. And of those who survived the first 4 weeks, 26.6/1000 died before their first birthday (table 3). The patterns of how mortality varies with duration of IPI are not as smooth as those for pregnancy outcomes, but they show that the risks of mortality are often higher for the shorter IPIs and lower for the longer IPIs. The percentage of babies known to be alive at 1 year is below the sample average for IPI $\leq$ 3 months and above the sample average for 3 months $<$ IPI $\leq 48$ months.

The patterns of how the unadjusted and adjusted RRRs of the outcome of the focal pregnancy vary with IPI duration are quite similar in our data and in the Love et al data on Scottish women (figure 1). In both studies, no significant effects of IPI duration are seen on the risks of a stillbirth, but the unadjusted relative risk of induced abortion increases monotonically as IPI duration increases, being lowest for IPI $\leq 6$ months (for Matlab unadjusted RRR for IPI $\leq 6$ months $=0.59,95 \%$ CI 0.40 to 0.86 , relative to $\mathrm{IPI}=6-12$ months) and highest for IPIs $>24$ months (for Matlab unadjusted RRR =3.07 (2.11

Table 3 Mortality after miscarriage in previous pregnancy, by interpregnancy interval (IPI) among all singleton live births $(n=8705)$

\begin{tabular}{|c|c|c|c|c|c|c|c|}
\hline \multirow{2}{*}{$\begin{array}{l}\text { IPI duration } \\
\text { (months) (\%) }\end{array}$} & \multicolumn{3}{|c|}{ Child's age at death } & \multirow{2}{*}{$\begin{array}{l}\text { Known alive } \\
\text { at } 1 \text { year }\end{array}$} & \multirow{2}{*}{$\begin{array}{l}\text { Migrated out } \\
\text { before age } 1\end{array}$} & \multirow[b]{2}{*}{ Total births } & \multirow[b]{2}{*}{ Column \% } \\
\hline & First week & Week 2-4 & Week 5-52 & & & & \\
\hline$\leq 3$ & $67(3.6)$ & $37(2.0)$ & $49(2.6)$ & 1647 (87.8) & $75(4.0)$ & $1875(100.0)$ & 21.5 \\
\hline $3-6$ & $64(3.1)$ & $26(1.3)$ & $54(2.6)$ & 1868 (90.1) & $62(2.9)$ & 2074 (100.0) & 23.8 \\
\hline $6-12$ & $81(3.3)$ & $28(1.1)$ & $75(3.1)$ & 2196 (89.6) & $72(2.9)$ & 2452 (100.0) & 28.2 \\
\hline $12-18$ & $31(3.9)$ & $8(1.0)$ & $13(1.6)$ & $714(90.0)$ & $27(3.4)$ & 793 (100.0) & 9.1 \\
\hline $18-24$ & $18(3.3)$ & $5(0.9)$ & $12(2.2)$ & 496 (91.3) & $12(2.2)$ & $543(100.0)$ & 6.2 \\
\hline 24-36 & $16(3.4)$ & $2(0.4)$ & $7(1.5)$ & 438 (93.4) & $16(3.4)$ & 469 (100.0) & 5.4 \\
\hline $36-48$ & $6(2.7)$ & $2(0.8)$ & $6(2.7)$ & 207 (91.5) & $5(2.2)$ & $226(100.0)$ & 2.6 \\
\hline$>48$ & 9 (3.3) & $2(0.7)$ & $4(1.5)$ & $243(89.0)$ & $15(5.5)$ & 273 (100.0) & 3.1 \\
\hline Total & $292(3.4)$ & $110(1.3)$ & $220(2.5)$ & 7799 (89.5) & $284(3.6)$ & 8705 (100.0) & \\
\hline Rate per 1000 at risk & 33.5 & 13.1 & 26.6 & & & & \\
\hline
\end{tabular}


1a. Abortion following Miscarriage by IPI (in months)

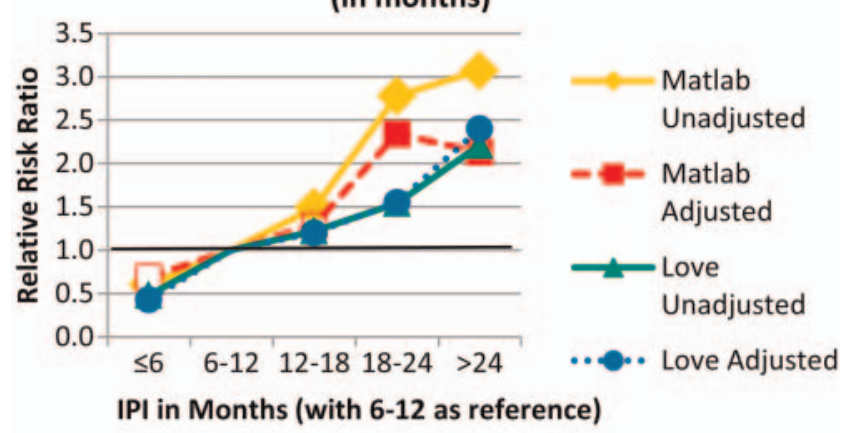

1b. Miscarriage following Miscarriage by IPI (in months)

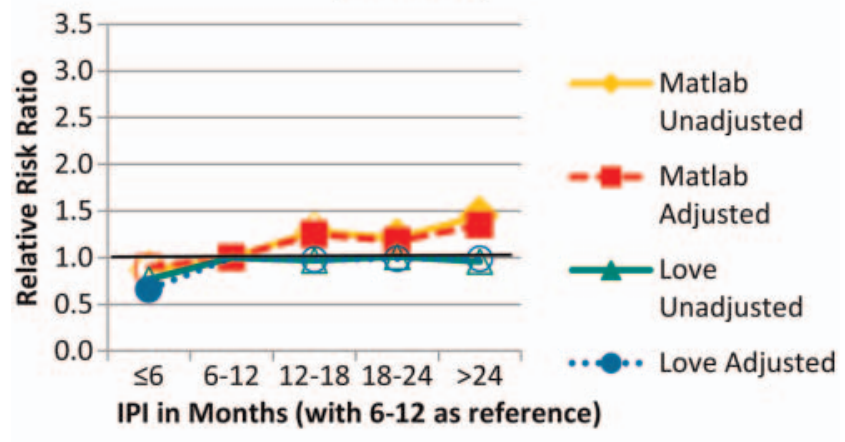

1c. Stillbirth following Miscarriage by IPI (in months)

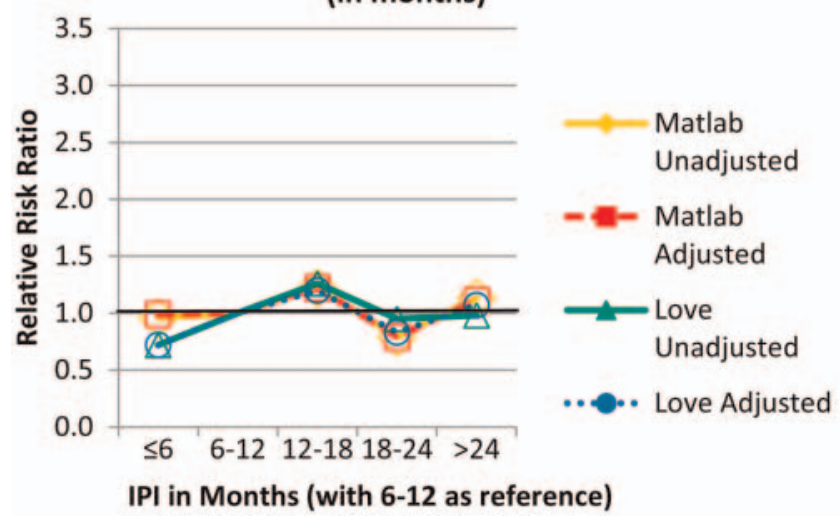

Figure 1 Relative risk ratios of induced abortion, miscarriage and stillbirth following a miscarriage by interpregnancy interval duration: unadjusted and adjusted results from Matlab and Love et $\mathrm{al}^{7}$ (Note: solid symbols indicate $\mathrm{p}<0.05$ ).

to 4.46 ) relative to $\mathrm{IPI}=6-12$ months). In Matlab, the unadjusted relative risks of a subsequent miscarriage also generally increase with IPI duration, being highest for IPIs $>24$ months, whereas in Scotland long IPIs were not associated with higher relative risks of miscarriage. For both induced abortion and miscarriage, the patterns are very similar in the two studies for the shortest IPIs, but the pernicious effects of long intervals on the unadjusted relative risks are larger for Matlab than in Scotland. Adjusting for other variables generally has more effect in our data from Matlab than it did in the Scottish data. In the Matlab data, the effect of adjustment is greatest for the longest intervals, so much so that the adjusted RRRs for IPIs $>24$ months on induced abortion are slightly lower for Matlab than for Scotland.

Unadjusted and adjusted RRRs of the focal-pregnancy outcome for our finer breakdown of IPI categories show that the same patterns exist within the $\mathrm{IPI} \leq 6$ and $>24$ months categories (figure 2), though the relative risk of a live birth for 24-36 months is lower than that for 18-24 months. Relative to IPI=6-12 months, pregnancies that were conceived $\leq 3$ months after a miscarriage were the most likely to result in a live birth and least likely to result in a miscarriage (adjusted RRR 0.70, $95 \%$ CI 0.59 to 0.86$)$ or induced abortion $(0.50,0.29$ to $0.89)$. Induced abortions were more likely following IPIs of $18-24$ months $(2.36,1.48$ to 3.75$)$, 36-48 months $(2.73,1.50$ to 4.94$)$, and $>48$ months $(3.32,2.05$ to 5.38$)$; and miscarriages were more likely following IPIs of 1217 months $(1.25,1.01$ to 1.56$)$ and $>48$ months (1.90, 1.40 to 2.58$)$. Again, adjustment has a greater effect the longer the IPI. Again, no significant effects of IPI duration are seen on the risks of a stillbirth.

Figure 3 shows the HR of mortality during the three subperiods of infancy for our finer breakdown of IPI categories. We find no significant relationships between IPI duration and early neonatal mortality in our unadjusted or adjusted analyses. However, for late neonatal mortality, in both the unadjusted and the adjusted analyses, we find significantly higher risk of mortality for IPIs $\leq 3$ months (adjusted HR 1.74 (1.06 to 2.84)) and generally see a decline in mortality as IPI duration increases up to 36 months. We find a significantly lower unadjusted risk of postneonatal mortality for IPIs of 12-18 months compared to those of 6-12 months $(0.54,0.30$ to 0.96$)$. The adjusted HR is similar but is not statistically significant $(0.56,0.31$ to 1.01$)$.

\section{DISCUSSION}

We find that the shorter the IPI following a miscarriage, the more likely the subsequent pregnancy is to result in a live birth. Women with IPI $>18$ months following a miscarriage, and especially those with $\mathrm{IPI}>48$ months have a much higher likelihood of experiencing another miscarriage or having an induced abortion. The relative risks of an induced abortion following a miscarriage are particularly high for the longest IPI category (unadjusted RRR for IPI $>48$ months $=5.02$ (3.13 to 8.03) and adjusted RRR $=3.32$ (2.05 to 5.38)). Adjusting for the effects of demographic and socioeconomic variables reduces the effect of long intervals on induced abortion, but they remain large and significant. No significant effects of IPI duration are seen on the risks of a stillbirth.

However, we see quite different patterns when we consider the effect of pregnancy spacing after a miscarriage on late neonatal and postneonatal mortality. Compared to IPIs of 6-12 months, the shortest IPIs following a miscarriage ( $\leq 3$ months) are associated with significantly higher unadjusted and adjusted HRs of late neonatal 
2a. Abortion following Miscarriage by IPI (in months)

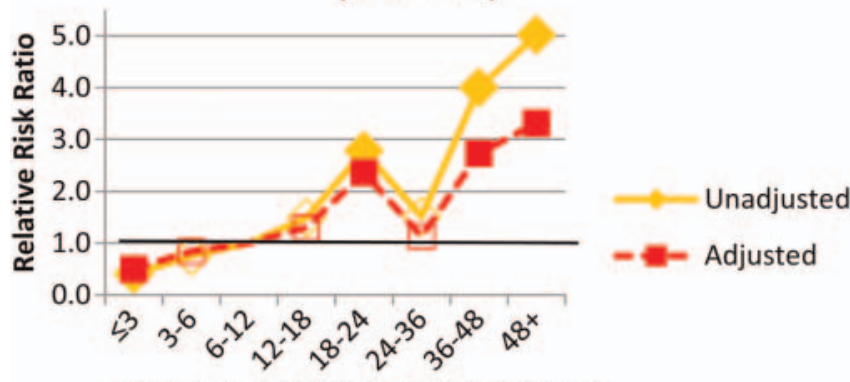

IPI in Months (with 6-12 as reference)

2b. Miscarriage following Miscarriage by IPI (in months)

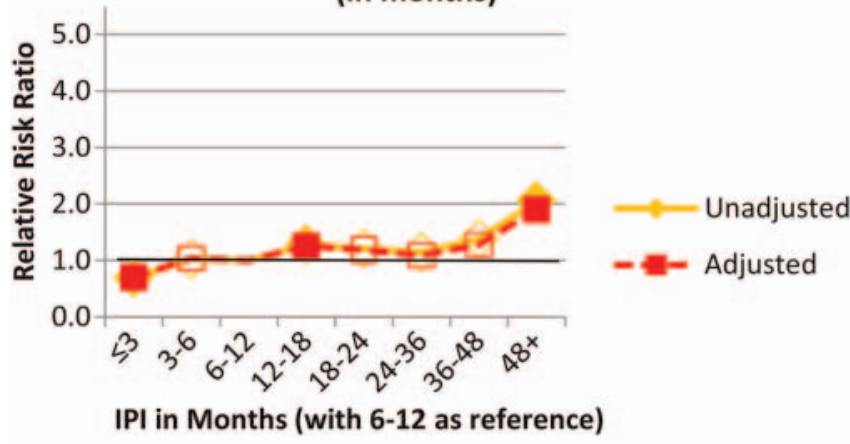

2c. Stillbirth following Miscarriage by IPI

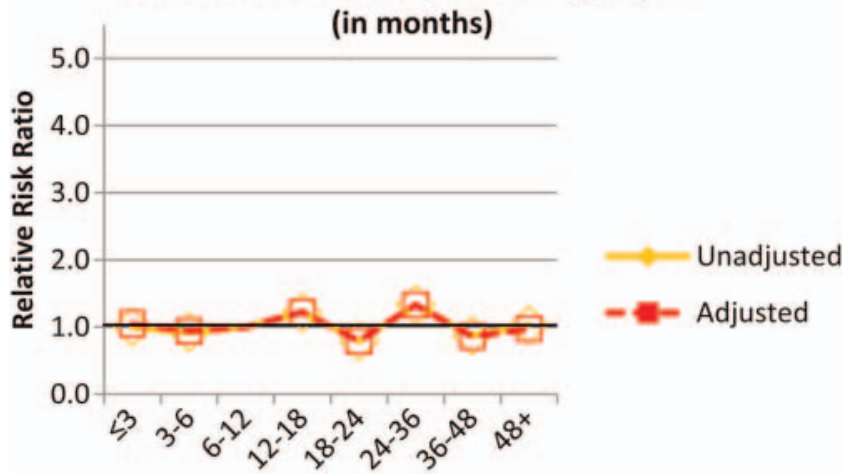

IPI in Months (with 6-12 as reference)

Figure 2 Relative risk ratios of induced abortion, miscarriage and stillbirth following a miscarriage by expanded interpregnancy interval categories: unadjusted and adjusted results for Matlab (Note: solid symbols indicate $p<0.05$ ).

mortality, and IPIs of 12-18 months are associated with a significantly lower unadjusted HR of postneonatal mortality. It appears that children born after very short IPIs following a miscarriage are able to survive the first week of life but then are at higher risk of dying in the rest of the first year.

\section{Comparison to other studies}

Most studies of the effects of pregnancy spacing consider intervals that began with a live birth or with a live birth or stillbirth. $^{1-3} 13$ They generally find adverse effects of both short and long intervals, but the 'optimum' interval (the

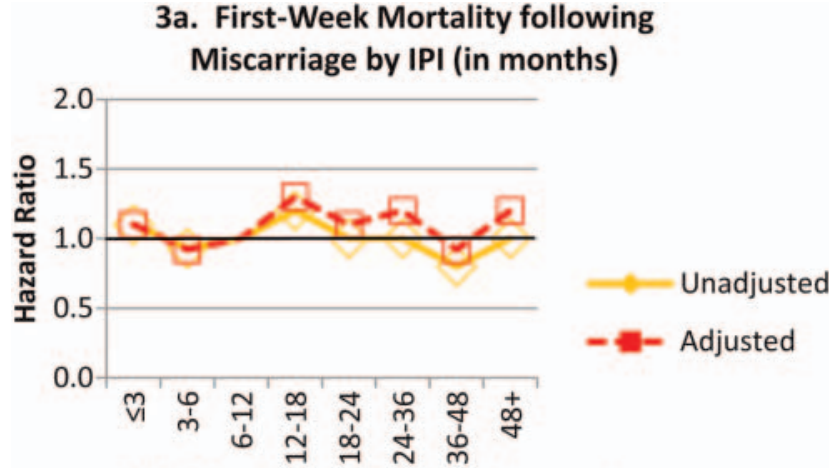

IPI in Months (with 6-12 as reference)

3b. Week 2-4 Mortality following Miscarriage by IPI (in months)

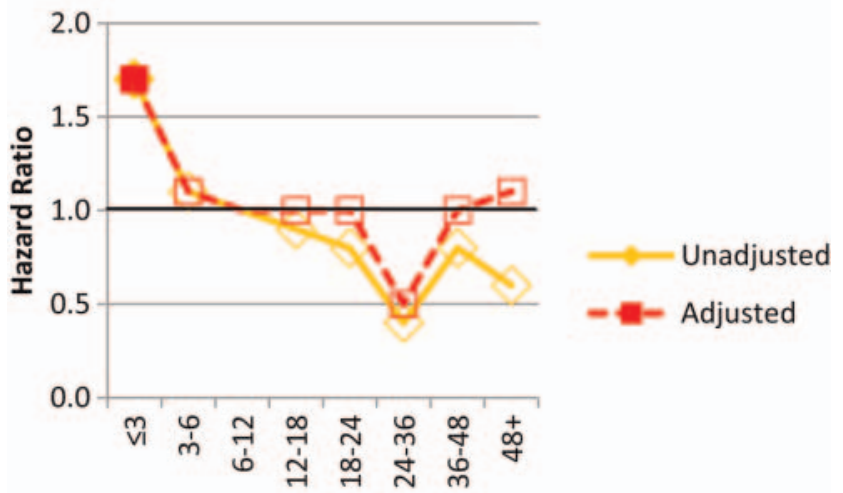

IPI in Months (with 6-12 as reference)

3c. Week 5-52 Mortality following Miscarriage by IPI (in months)

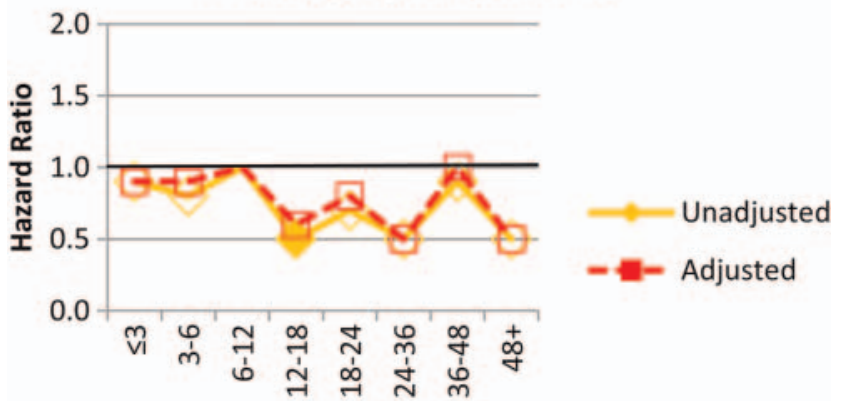

IPI in Months (with 6-12 as reference)

Figure 3 HR of mortality during subperiods of infancy, by interpregnancy interval duration, unadjusted and adjusted results from Matlab (Note: solid symbols indicate $p<0.05$ ).

one with the lowest risk of an adverse outcome) differs across types of outcomes. For example, a study of the USA that considers intervals that began with live births finds the lowest risks of adverse perinatal outcomes for IPIs of $18-23$ months duration. ${ }^{14} \mathrm{~A}$ meta-analysis of the effects of intervals following live births on perinatal outcomes found that intervals of 18-59 months are associated with better outcomes than shorter and longer intervals, ${ }^{2}$ and a review of studies of maternal outcomes reaches a similar conclusion. ${ }^{1}$ An analysis of data from a 
number of developing countries found infant mortality to be lowest for intervals $>24$ months duration that began with live births, and under-five mortality to be lowest for intervals $>36$ months. ${ }^{3}$

A study of the Matlab MCH-FP Area found that following live births the risks of miscarriage and of stillbirth in the next pregnancy were significantly higher for IPIs $\leq 6$ months (compared to those of 27-50 months duration). ${ }^{12}$ That study did not distinguish the type of outcome that began IPIs $>50$ months. An earlier study in Bangladesh found a higher risk of early fetal death (first or second trimester) following short IPIs ( $<12$ months) that began with the birth of a surviving child who breastfed. ${ }^{15}$ Studies using data from Sweden found that very short ( $\leq 3$ months) IPIs following live births were associated with higher risks of stillbirth. ${ }^{16}{ }^{17}$ Studies of World Fertility Survey data from a number of developing countries found IPIs $<9$ months following live births to be associated with higher risks of fetal death; ${ }^{18} 19$ early fetal losses and stillbirths were combined in those studies.

Very few studies have looked specifically at IPIs that began with a miscarriage, as we do here. A study of Latin America that assessed the effects of intervals following induced and spontaneous abortions found that intervals $<6$ months between abortion and subsequent pregnancy were associated with elevated risks of premature rupturing of membranes, anaemia, bleeding, preterm and very preterm births, and low birthweight, compared with longer intervals. ${ }^{20}$ However, that study did not distinguish between induced and spontaneous abortions. There are reasons to expect that the effects might differ considerably for the two-one being a voluntary termination of a pregnancy that was most likely unintended and the other being the unexpected termination of a pregnancy that was more likely to have been intended. Based on the study of Latin America just mentioned, WHO currently recommends 'After a miscarriage or induced abortion, the recommended minimum interval to next pregnancy should be at least 6 months in order to reduce risks of adverse maternal and perinatal outcomes'. ${ }^{21}$ The report on the WHO Technical Consultation that makes that recommendation comments 'More studies on the effects of postabortion pregnancy intervals are needed in different regions. A distinction between induced and spontaneous abortion ... would be particularly helpful in future studies' (p. 3). ${ }^{21}$

Three studies ${ }^{4-6}$ using data from the USA or Europe found no effects of the duration of IPI following a miscarriage on the outcome of the subsequent pregnancy, but their samples were relatively small (64, 91 and 1530, respectively). An earlier study of Matlab that considered a much smaller sample of IPIs that began with a miscarriage than that considered here and only in the MCH-FP Area also found, as we do here, a decreasing likelihood of having a live birth following a miscarriage as duration of the preceding IPI increases. ${ }^{12}$ However, that study did not consider longer intervals that began with a miscarriage.
Love $e t a l \mathrm{~s}^{7}$ recent study used a large sample of pregnancies to Scottish women who had a miscarriage to assess the effects of pregnancy spacing on the outcome of the subsequent pregnancy. We have constructed our analyses to be as similar as possible to those of Love $e t a l$, to facilitate comparisons. Our results for pregnancy outcomes are remarkably similar to theirs. Both studies find that short IPIs following a miscarriage are associated with lower risks of a subsequent miscarriage or an induced abortion, and long intervals are associated with higher risks of these outcomes, and both find no significant effects of the duration of the postmiscarriage IPI on the risk of stillbirth.

We also examine even shorter and longer IPIs durations than Love et al do and show that the very shortest intervals we consider ( $\leq 3$ months) are associated with the lowest risks of induced abortion and miscarriage and the longest ( $>48$ months) are associated with the highest risks of these outcomes.

We generally find even stronger pernicious effects of long intervals on the relative risk of a miscarriage or an induced abortion in the focal pregnancy than was found for Scottish women, and the effects are particularly large when we consider an expanded set of IPI categories (up to $>48$ months). Adjusting for the effects of demographic and socioeconomic variables reduces the effects of long intervals on the likelihood of induced abortion more for Matlab than it did in Love et als study of Scotland; the adjusted ORs associated with IPI $>24$ months (compared to that of 6-12 months) is slightly lower for Matlab than that Love et al found for Scotland (whereas the opposite is true for unadjusted ORs). The Love et al study only considers cases where the miscarriage that began the IPI was the first recorded pregnancy outcome for the woman, whereas we consider all IPIs that began with a miscarriage and control for gravidity in our analyses. This may be one reason why we find greater effects of controlling for other variables than they do. In our data there are 2461 first pregnancies that ended with a miscarriage. We conducted our analysis for this subsample and found patterns similar to those reported here, but they were not statistically significant.

We find some evidence that short IPIs following miscarriages are associated with higher mortality between the first week and the end of the first year of life for the children born after a miscarriage. Another study of Matlab found that short interoutcome intervals $(<15$ months between one pregnancy outcome and the next outcome) that began with a miscarriage were associated with higher risks of early and late neonatal mortality compared with intervals of 36-59 months that began with the live birth of a child who survived. ${ }^{22}$ However, that study did not compare them to longer intervals that began with a miscarriage. In contrast, Love et al do not find short IPIs to be associated with higher risks of preterm delivery and low birthweight—outcomes that have been widely found to be associated with 
mortality during infancy. ${ }^{23} 24$ The better nutritional status of Scottish women may buffer their fetuses from the depleting effects of a recent previous miscarriage.

Previous studies have offered a number of hypotheses to explain why there might be adverse effects of short IPIs, the main ones being (1) competition for family resources and time from a just-older sibling; ${ }^{22}$ (2) transmission of infection among closely spaced siblings; ${ }^{22}$ and (3) maternal depletion, ${ }^{25}$ especially of folate ${ }^{26}$ The first and second mechanisms would only come into play for intervals that began with live births of children who survived, and hence do not apply to IPIs that began with miscarriages. Maternal depletion is more likely the longer the pregnancy. ${ }^{25}$ Folate depletion begins around 5 months gestation. ${ }^{26}$ Since our definition of miscarriages includes pregnancies up to 28 weeks gestation, some of the pregnancies could lead to folate depletion. Our results for infant mortality (but not for pregnancy outcomes) are consistent with the idea that pregnancies that result in miscarriages deplete vital nutrients and that women require time to replete them in order to give birth to a healthy child that will survive its first year. Our finding of a pernicious effect for children but not for women is consistent with studies that show that the effects of maternal depletion can be different for the mother and the fetus, with the fetus being affected more than the mother in cases of severe nutritional deficiencies. ${ }^{27}$

Our finding that short IPIs following a miscarriage are associated with a greater likelihood of a live birth at the end of the interval is consistent with the notion that most women who had a miscarriage wanted to have a live birth, and as a result many of them seek to become pregnant again as soon as possible and may take very good care of themselves during the subsequent pregnancy. A fifth $(20.5 \%)$ of the women in our sample who experienced a miscarriage and became pregnant again did so within 3 months of the miscarriage, and $44 \%$ were pregnant within 6 months.

To explain the adverse effects of long IPIs on pregnancy outcomes, it has been hypothesised that one pregnancy prepares the woman's body for the next and that this 'protection' decreases as time passes, making pregnancies following long intervals similar to first pregnancies, ${ }^{14}$ which have been shown to have higher risk of many poor outcomes. ${ }^{28}$ It is also possible that long intervals are selective of women in poorer health, who take longer to conceive ${ }^{29}$ or that women who have long intervals did not want to become pregnant again and do not take as good care of themselves during pregnancy. ${ }^{12}$ In addition, long IPIs are more likely for older women; older maternal age is associated with its own independent adverse effects on pregnancy outcomes, ${ }^{30}$ though we see an effect even when we control for maternal age. A meta-analysis has shown that IPIs longer than 59 months are associated with adverse perinatal outcomes. ${ }^{2}$ That study also found adverse effects on perinatal outcomes of intervals shorter than 18 months, which we do not see for pregnancy outcomes, but we do see some adverse effects of very short intervals on infant survival. Other studies of Matlab have shown that women with long intervals (but not distinguishing the type of outcome with which they began) have higher risks of pregnancy complications, ${ }^{31}$ maternal mortality $^{29}$ and induced abortion. ${ }^{12}$

\section{Strengths and weaknesses of the study}

We look at the effects of IPIs following miscarriages, allowing conclusions about how long women should wait after a miscarriage before becoming pregnant again. We replicate the Love et $a l^{7}$ study, which also looked at this question, in a very different setting-poor women in rural Bangladesh. Furthermore, we examine the effects of shorter and longer intervals than considered by Love et al. We consider recent data (up to 2008)-more recent than considered by Love et al (1981-2000).

The Matlab DSS data on induced abortion and miscarriage are likely to be of high quality and not to suffer from underreporting. In their many years of work in the community the CHWs have established themselves as trustworthy and in a good position to collect reliable information on pregnancy outcomes and, because of their frequent household visits, they are likely to elicit accurate information. ${ }^{9}$ Nonetheless, there is probably an under-reporting of early miscarriages since these may not have been identified as pregnancies, and there may be some underreporting of induced abortions and some misreporting of these as miscarriages. Furthermore, the gestation of pregnancy is based on women's reports of the DLMP, rather than on sonography, which is very rare in Matlab. The reports of DLMP, however, are likely to be quite accurate, since the respondents were visited regularly and the recall periods were relatively short.

The DSS defines a stillbirth as a fetal loss at 28 weeks or longer gestation and miscarriage as a spontaneous fetal loss prior to 28 weeks. Some studies define stillbirth starting at 20 weeks (and Love et al use a 24-week cutoff), so their definition of stillbirth overlaps with our definition of miscarriage. In our data, for cases for which we know DLMP, there were 50 (of 578) cases where the focal outcome was coded as a miscarriage and the duration of gestation was 20-27 weeks. We are not able to recode these cases, however, because we do not know pregnancy duration for cases for which DLMP is not reported and must rely on the reported outcome of pregnancy for those cases. The fact that we find no evidence of maternal depletion on pregnancy outcomes even with a miscarriage definition of 28+ weeks suggests that we would not have seen one had we been able to use a 20+-week or 24+-week definition.

Though smaller than the sample used by Love et al., our sample $(n=10435)$ is much larger than that used in other studies of this topic. ${ }^{4-612}$

Love $e t$ al found a positive association of the duration of the IPI with the incidence of ectopic pregnancy, caesarean section, preterm delivery and low birthweight. We either do not have these indicators in our data or have 
them only for a subsample too small to permit analyses. However, unlike Love et al, for IPIs that end in live births, we look at the mortality of those children during three subperiods of infancy.

We do not consider some possibly confounding variables, for example, use and quality of prenatal care and the woman's health and fecundity, which may affect the outcomes of interest and could illuminate the mechanisms underlying the effects we find.

\section{Implications for research}

This study is of a setting, rural Bangladesh, where fertility and infant mortality rates are relatively high but have fallen considerably over the study period, and one half of the area studied has been exposed to more intense, higher-quality family planning services than are available in many developing countries. The study should be replicated in other settings. Future studies should adjust for the effects of additional potentially confounding variables and assess the effects of the durations of IPIs following miscarriages on the health and survival of the children born at the end of those intervals as well as on those of their mothers. Studies should also assess the effects of IPIs that began with stillbirths and of IPIs that began with induced abortions.

\section{Implications for clinical practice}

The current WHO recommendation is that women should wait at least 6 months after a miscarriage or induced abortion before becoming pregnant again. However, as noted above, that recommendation was based on one study of Latin America of the effects of IPIs following induced or spontaneous abortions. ${ }^{20}$ Our study, of Matlab, Bangladesh, like that of Love $e t a l^{7}$ for Scotland, other studies of industrialised countries, ${ }^{4-6}$ and a smaller study of Matlab, ${ }^{12}$ looks specifically at the effects of IPIs following miscarriages; all the studies find no higher risk of adverse pregnancy outcomes if women become pregnant soon after a miscarriage. However, we find that very short intervals ( $\leq 3$ months) following a miscarriage are associated with higher mortality risks for infants in Bangladesh, which suggests that, for the sake of child survival, in less developed settings it may be best for women to wait to at least 3 months before becoming pregnant again following a miscarriage. Steer noted a similar concern in a 2007 editorial in BJOG. ${ }^{32}$

In developed settings, such as that considered in the Love et al study, there is concern that postponing pregnancies after miscarriages may lead to difficulties in conceiving and greater probabilities of miscarriage because of older women's age. This is less of a concern in poor countries such as Bangladesh, where women begin (and often end) childbearing at earlier ages than in more developed countries.

\footnotetext{
Author affiliations

${ }^{1}$ Labor and Population Unit, The RAND Corporation, Santa Monica, California, USA
}

${ }^{2}$ Department of Preventive Medicine, Stony Brook University, Stony Brook, New York, USA

${ }^{3}$ MEASURE Evaluation, University of North Carolina, Chapel Hill, North Carolina, USA, and International Centre for Diarrhoeal Disease Research, Bangladesh, Dhaka, Bangladesh

Acknowledgements We thank Sohinee Bhattacharya, Katherine J Gold, Jayne Lucke, Maureen Norton and Shea Rutstein for their comments on an earlier draft of this paper and Abdur Razzaque for helping with the construction of the data file.

\section{Collaborators Abdur Razzaque.}

Contributors JD conceived the study, oversaw the data analysis, and wrote the paper. LH conducted the data analysis and assisted with the writing of the paper. MR designed the data file construction and assisted with the writing of the paper.

Funding Support for the research was provided by the Office of Population and Reproductive Health, Bureau for Global Health, US Agency for International Development under the terms of Cooperative Agreement No. GPO-A-00-05-00027-00 awarded to the Extending Service Delivery (ESD) Project. ESD is a partnership between Pathfinder International (where MR was employed when the study began); IntraHealth International, Inc.; Management Sciences for Health; and Meridian Group International, Inc. Pathfinder issued a subcontract to icddr,b, where MR is currently employed. JD and LH worked on this research as consultants to Pathfinder. The views expressed are those of the authors and do not reflect the opinions of the funding agencies or the institutions with which the authors are affiliated.

\section{Competing interests None.}

Ethics approval Formal ethical review was not necessary for this study because only anonymised data were analysed. The data file was created based on records of the Matlab Demographic Surveillance System (DSS) of icddr,b. DSS data collection and management procedures are approved by the icddr,b Ethical Review Committee.

Provenance and peer review Not commissioned; externally peer reviewed.

Data sharing statement No additional data are available. Permission of icddr, b may be sought to use Matlab DSS data for specific research questions.

\section{REFERENCES}

1. Conde-Agudelo A, Rosas-Bermúdez A, Kafury-Goeta AC. Effects of birth spacing on maternal health: a systematic review. AJOG 2007; 196:297-308.

2. Conde-Agudelo A, Rosas-Bermúdez A, Kafury-Goeta AC. Birth spacing and risk of adverse perinatal outcomes: a meta-analysis. JAMA 2006;295:1809-23.

3. Rutstein SO. Further evidence of the effects of preceding birth intervals on neonatal, infant, and under five-years mortality and nutritional status in developing countries: evidence from the Demographic and Health Surveys. DHS Working Paper No. 41, Calverton, MD: Macro International Inc, 2008.

4. Goldstein RR, Pruyn MA, Croughan MS, et al. Neonatal outcomes in immediate versus delayed conceptions after spontaneous abortion: a retrospective case series. AJOG 2002;186:1230-6.

5. Rud B, Klunder K. The course of pregnancy following spontaneous abortion. Acta Obstet Gynecol Scand 1985;64:277-8.

6. Wyss P, Biedermann K, Huch A. Relevance of the miscarriagenew pregnancy interval. J Perinat Med 1994;22:235-41.

7. Love E, Bhattacharya S, Smith $\mathrm{N}$, et al. Effect of interpregnancy interval on outcomes of pregnancies after miscarriage: retrospective analysis of hospital episode statistics in Scotland. BMJ 2010;341: c3967.

8. National Institute of Population and Research and Training (NIPORT), Mitra Associates, ORC Macro. Bangladesh demographic and health survey 2007. Dhaka, Bangladesh, and Calverton, Maryland, USA, 2009.

9. D'souza S. A population laboratory for studying disease processes and mortality-the Demographic Surveillance System, Matlab, Comilla, Bangladesh. Rural Demogr 1981;8:29-51.

10. Fauveau V. Matlab: women, children, and health. Dhaka, Bangladesh: International Centre for Diarrhoeal Disease Research (icddr,b), 1992. 
11. Van Ginneken J, Bairagi A, De Francisco A, et al. Health and demographic surveillance in Matlab: past, present, and future. Scientific Publication, No. 72. Dhaka, Bangladesh: International Centre for Diarrhoeal Disease Research, 1998.

12. DaVanzo J, Hale L, Razzaque A, et al. Effects of interpregnancy interval and outcome of the preceding pregnancy on pregnancy outcomes in Matlab, Bangladesh. BJOG 2007;114:1079-87.

13. Conde-Agudelo A, Belizán JM. Maternal morbidity and mortality associated with interpregnancy interval: cross sectional study. BMJ 2000;321:1255-9.

14. Zhu BP, Rolfs RT, Nangle BE, et al. Effect of the interval between pregnancies on perinatal outcomes. N Engl J Med 1999;340:589-94.

15. Swenson I, Harper PA. The relationship between fetal wastage and pregnancy spacing in Bangladesh. Soc Biol 1978;25:251-7.

16. Stephansson O, Dickman PW, Cnattingius S. The influence of interpregnancy interval on the subsequent risk of stillbirth and early neonatal death. Obstet Gynecol 2003;102:101-8.

17. Smith GC, Pell JP, Dobbie R. Interpregnancy interval and risk of preterm birth and neonatal death: retrospective cohort study. BMJ 2003;327:313.

18. Casterline JB. Collecting data on pregnancy loss: a review of evidence from the World Fertility Survey. Stud Fam Plann 1989;20:81-95.

19. Casterline JB. Maternal age, gravidity, and pregnancy spacing effects on spontaneous fetal mortality. Soc Biol 1989;36:186-212.

20. Conde-Agudelo A, Belizán JM, Breman R, et al. A. Effect of the interpregnancy interval after an abortion on maternal and perinatal health in Latin America. IJGO 2005;89(Suppl 1):S34-40.

21. World Health Organization. Report of a WHO technical consultation on birth spacing. Geneva, Switzerland 13-15 June 2005. http://www. who.int/maternal_child_adolescent/documents/birth_spacing.pdf

22. DaVanzo J, Hale L, Razzaque A, et al. The effects of pregnancy spacing on infant and child mortality in Matlab, Bangladesh: how they vary by the type of pregnancy outcome that began the interval. Popul Stud 2008;62:131-54.

23. Yasmin S, Osrin D, Paul E, et al. Neonatal mortality of low-birth-weight infants in Bangladesh. Bull World Health Organ 2001;79:607-14.

24. McCormick MC. The contribution of low birth weight to infant mortality and childhood morbidity. N Engl J Med 1985;312: 82-90.

25. Winkvist A, Rasmussen KM, Habicht JP. A new definition of maternal depletion syndrome. Am J Pub Health 1992;82:691-4.

26. Smits LJ, Essed GG. Short interpregnancy intervals and unfavourable pregnancy outcome: role of folate depletion. Lancet 2001;358:2074-7.

27. King JC. The risk of maternal nutritional depletion and poor outcomes increases in early or closely spaced pregnancies. J Nutr 2003;133(5 Suppl 2):1732S-6S.

28. Yudkin PL, Baras M. A new approach to assessing the effect of birth order on the outcome of pregnancy. J Biosoc Sci 1983;15:307-16.

29. Rahman M, DaVanzo J, Razzaque A, et al. Demographic, programmatic, and socioeconomic correlates of maternal mortality in Matlab, Bangladesh. Pathfinder International Research and Evaluation Working Paper, July 2009; http://www.pathfind.org/site/ DocServer/

ME_Working_Paper_Correlates_of_Maternal_Mortality_-_for_.pdf? docID=15641

30. Nybo Andersen AM, Wohlfahrt J, Christens P, et al. Maternal age and fetal loss: population based register linkage study. BMJ 2000;320:1708-12.

31. Razzaque A, DaVanzo J, Rahman M, et al. Pregnancy spacing and maternal morbidity in Matlab, Bangladesh. IJGO 2005;89:541-9.

32. Steer P. Getting pregnant again too quickly (Editor's choice). BJOG 2007; 114 :i-ii. 